\title{
Defaults and lexical prototypes
}

Workshop on defaults in morphological theory

May 21, 2012

Rob Malouf

San Diego State University 


\section{Head-Driven Phrase Structure}

Representations in HPSG are typed feature structures, a class of directed acyclic graphs

An attribute value matrix is a description which picks out a sets of these linguistic objects

Each feature structure has a type associated with it

Types are organized into a signature which specifies appropriateness and inheritance relationships 


\section{Type hierarchies}

Types are organized into an inheritance hierarchy, an ontology of object types

The hierarchy is a bounded complete partial order: every pair of types have a unique least upper bound and there is a unique most-general-type

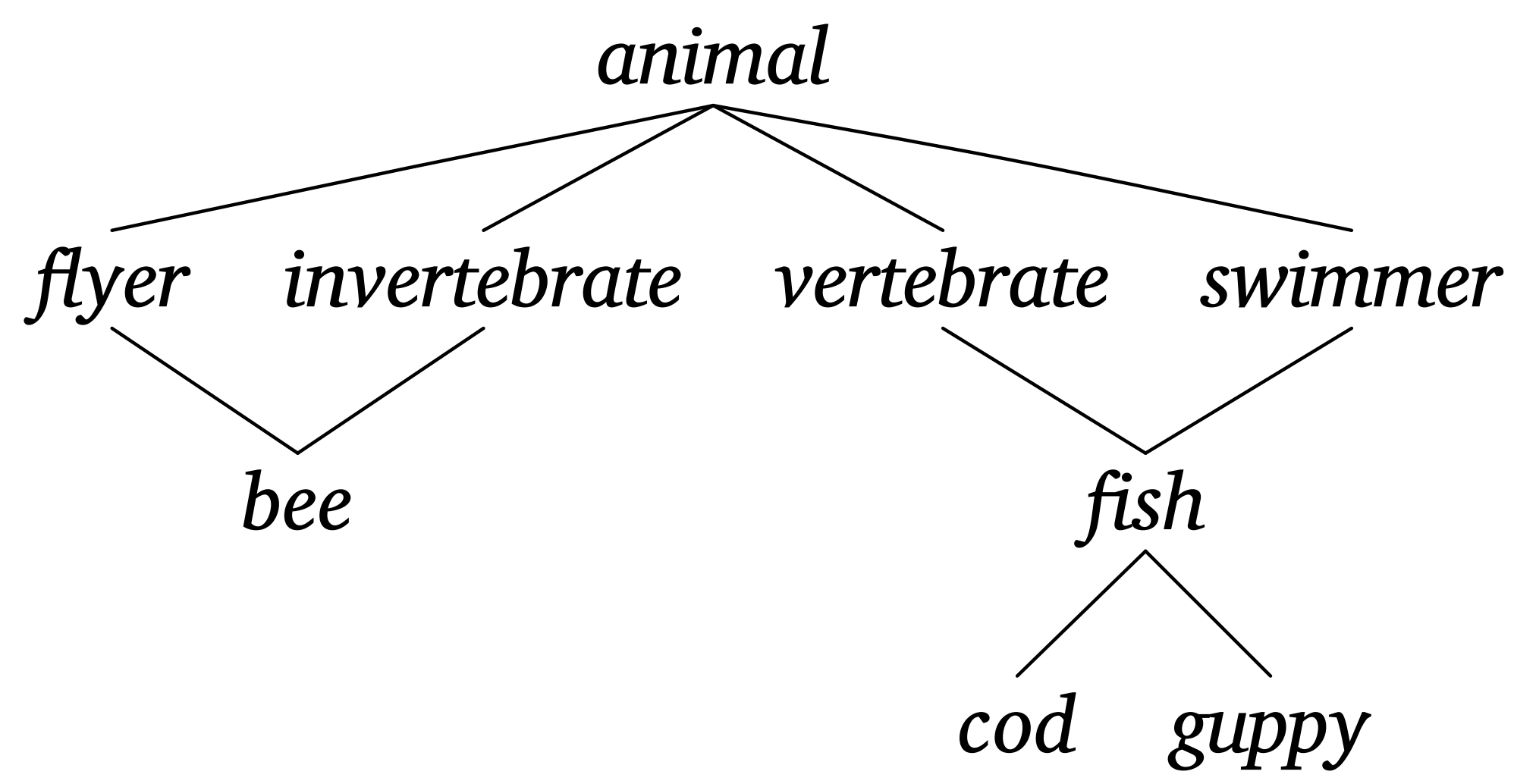




\section{Sort hierarchies}

The inheritance hierarchy defines an ontology of linguistic objects (sorts):

types and their relations ('is a' and 'has a')

appropriate features

appropriate values

type inference

Provides a basis for precise and efficient implementation (Flickinger 2000)

This ontology is (mostly) arbitrary and (mostly) universal

This metalanguage is important but not by itself linguistically very interesting 


\section{Sort hierarchies}

Grammar Matrix (Bender, et al. 2010)

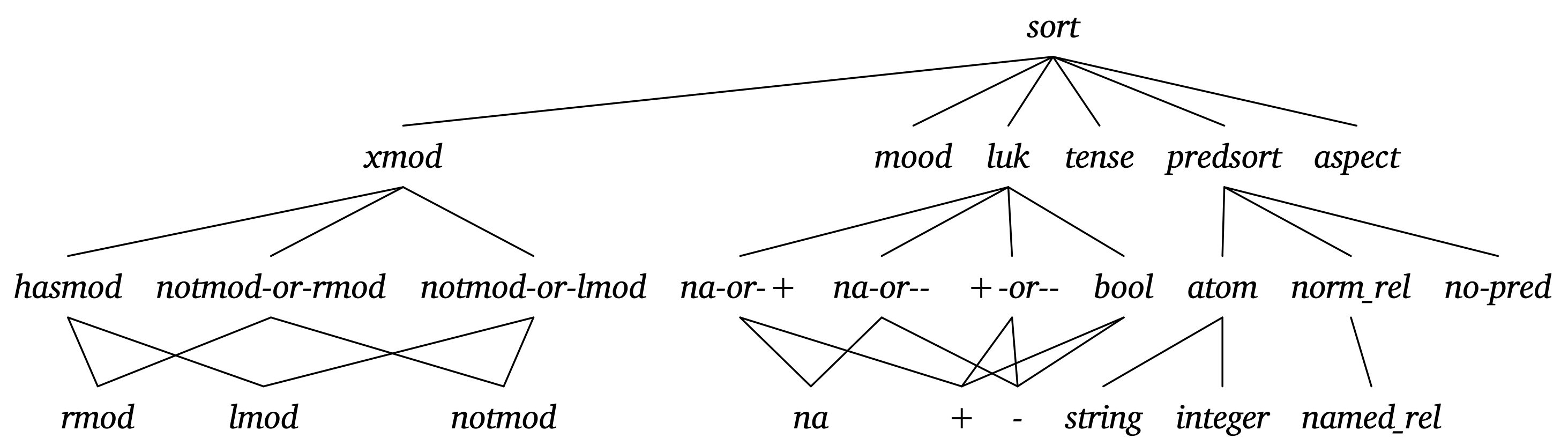




\section{Sort hierarchies}

Grammar Matrix (Bender, et al. 2010)

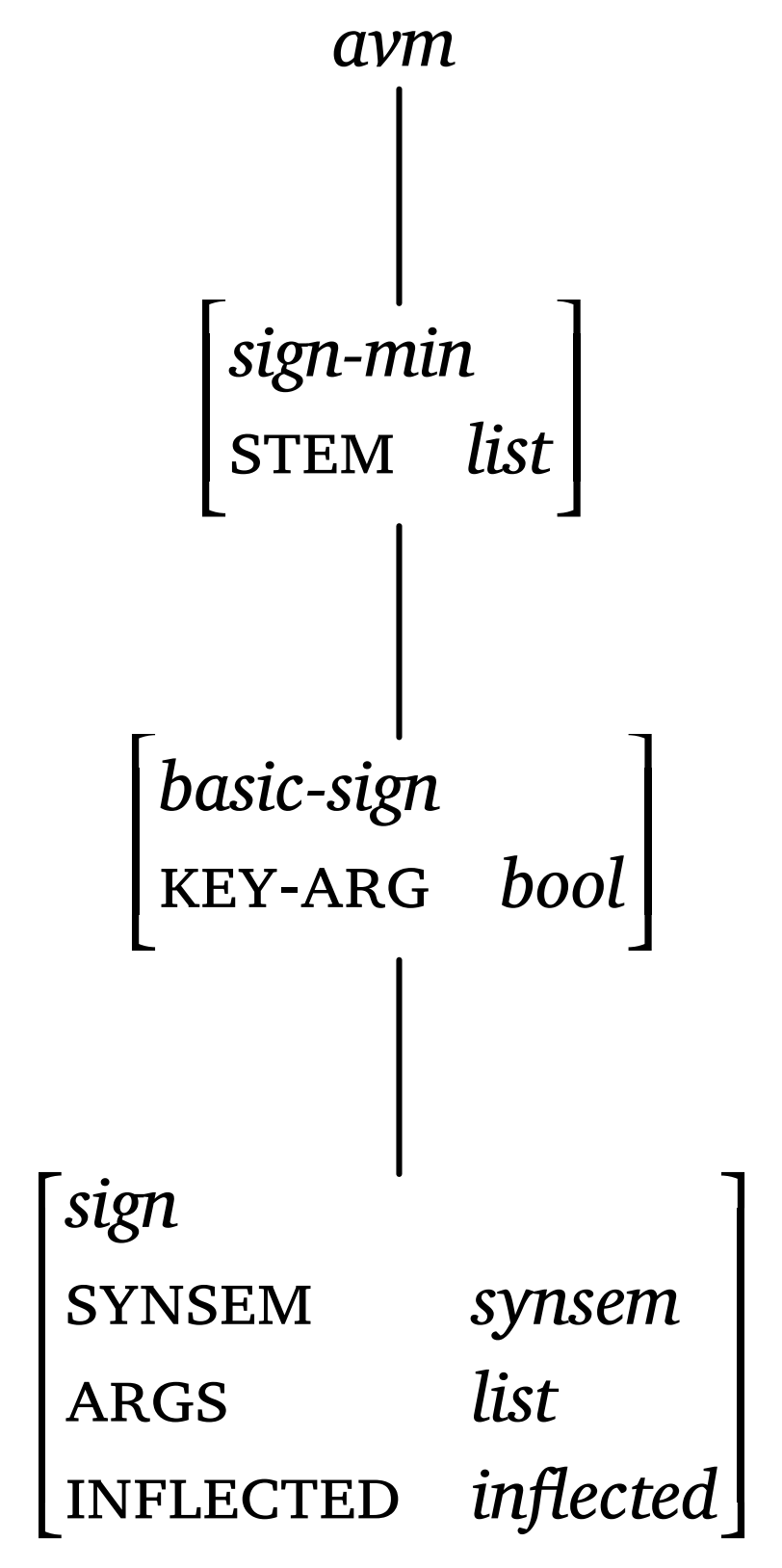




\section{Lexical hierarchies}

The type hierarchy is also used to define constraints on the lexicon and the inventory of constructions

Classes of words can be the same in some ways and different in others

Patterns of sameness can be reified as super-types, while differences are instantiated on lower types in the hierarchy

Anything that is true of a type is also true of all of any more specific type

Taxonomic approach to linguistic description 


\section{Lexical hierarchies}

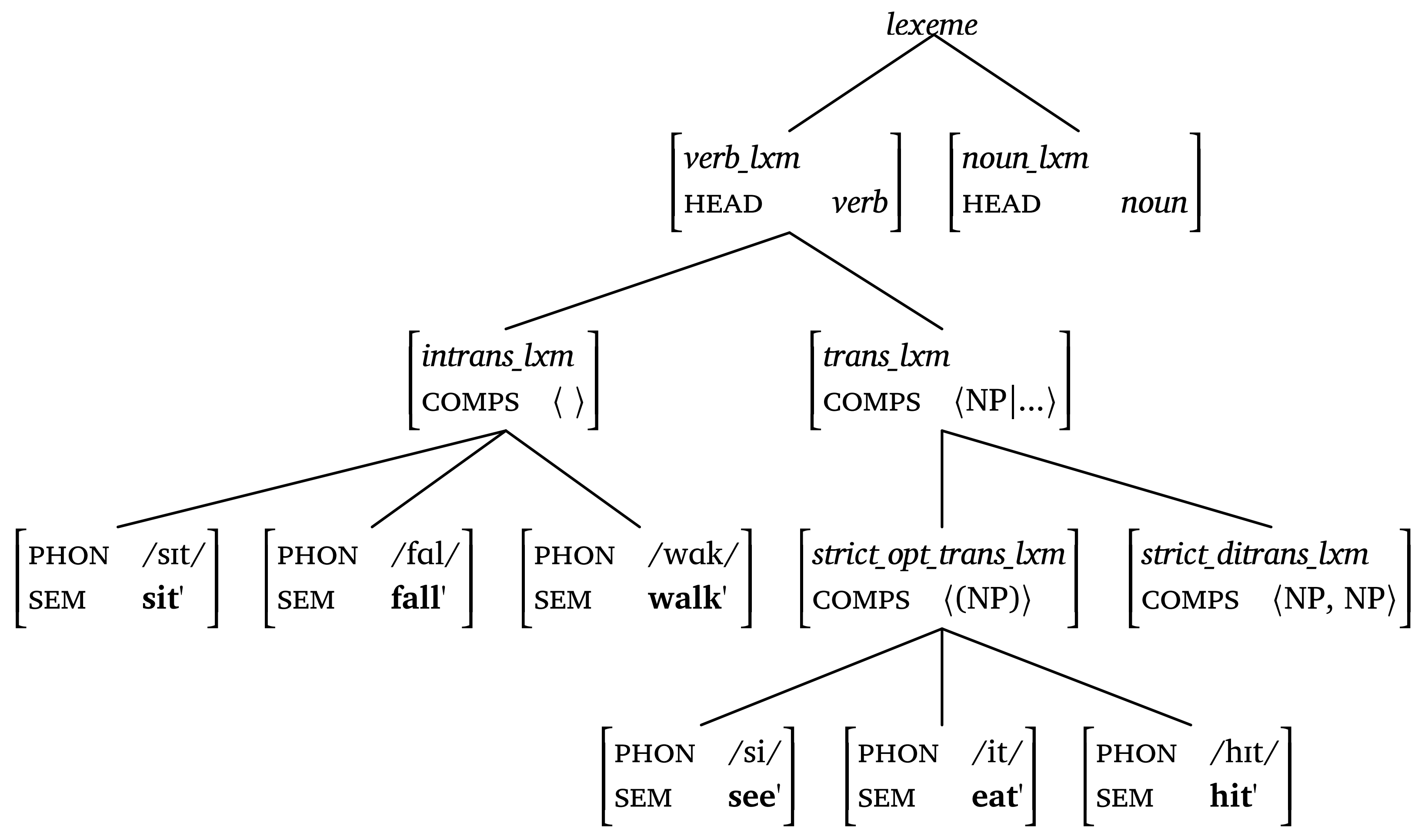




\section{Lexical hierarchies}

This style of representation associates patterns of sameness and differentness with particular types

Radial / family resemblance categories (Wittgenstein, Rosch, Lakoff, et al.) pose a problem

\begin{tabular}{c|ccc} 
& F & G & H \\
\hline$a$ & + & + & - \\
$b$ & + & - & + \\
$c$ & - & + & +
\end{tabular}

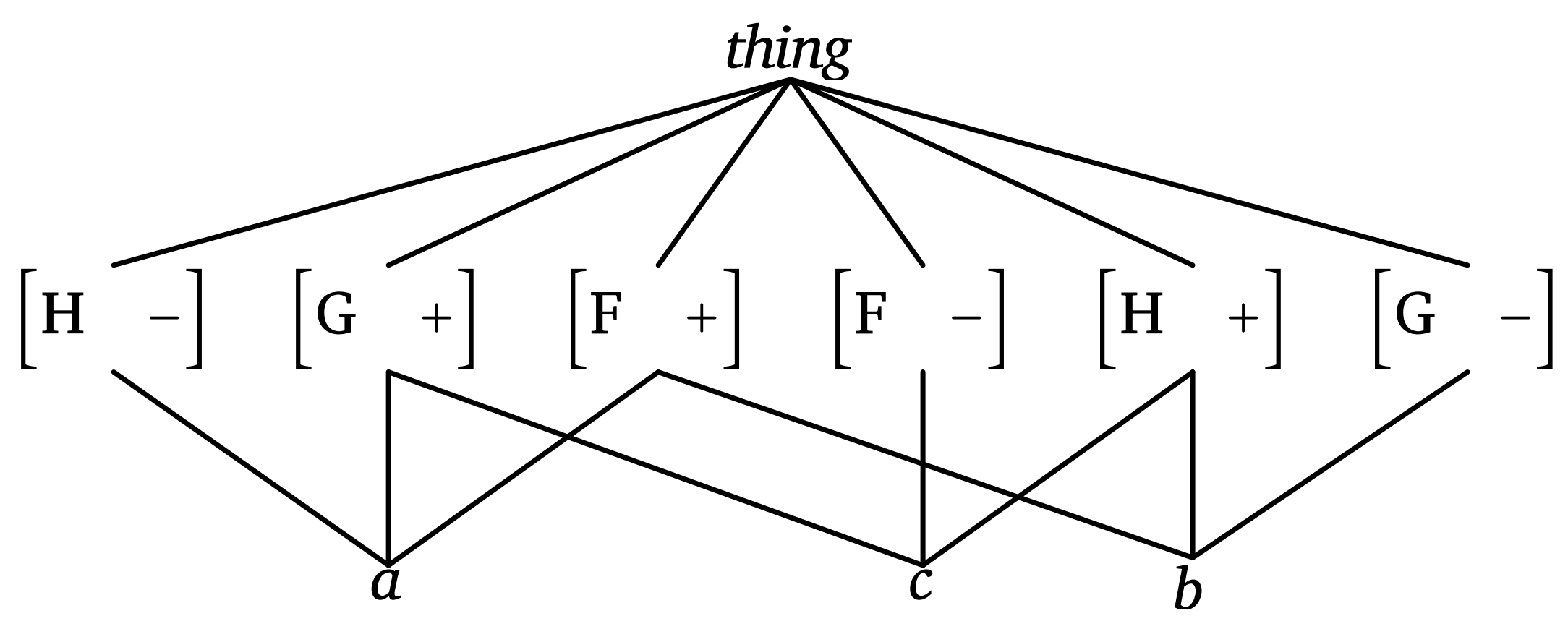




\section{Default inheritance}

Default constraints offer a solution to this problem

We can state properties of a type which usually hold, but allow more specific subtypes to override that

Anything that is true of a type is also true of all of any more specific type unless there's a conflict

\begin{tabular}{c|ccc} 
& F & G & H \\
\hline$a$ & + & + & - \\
$b$ & + & - & + \\
$c$ & - & + & +
\end{tabular}

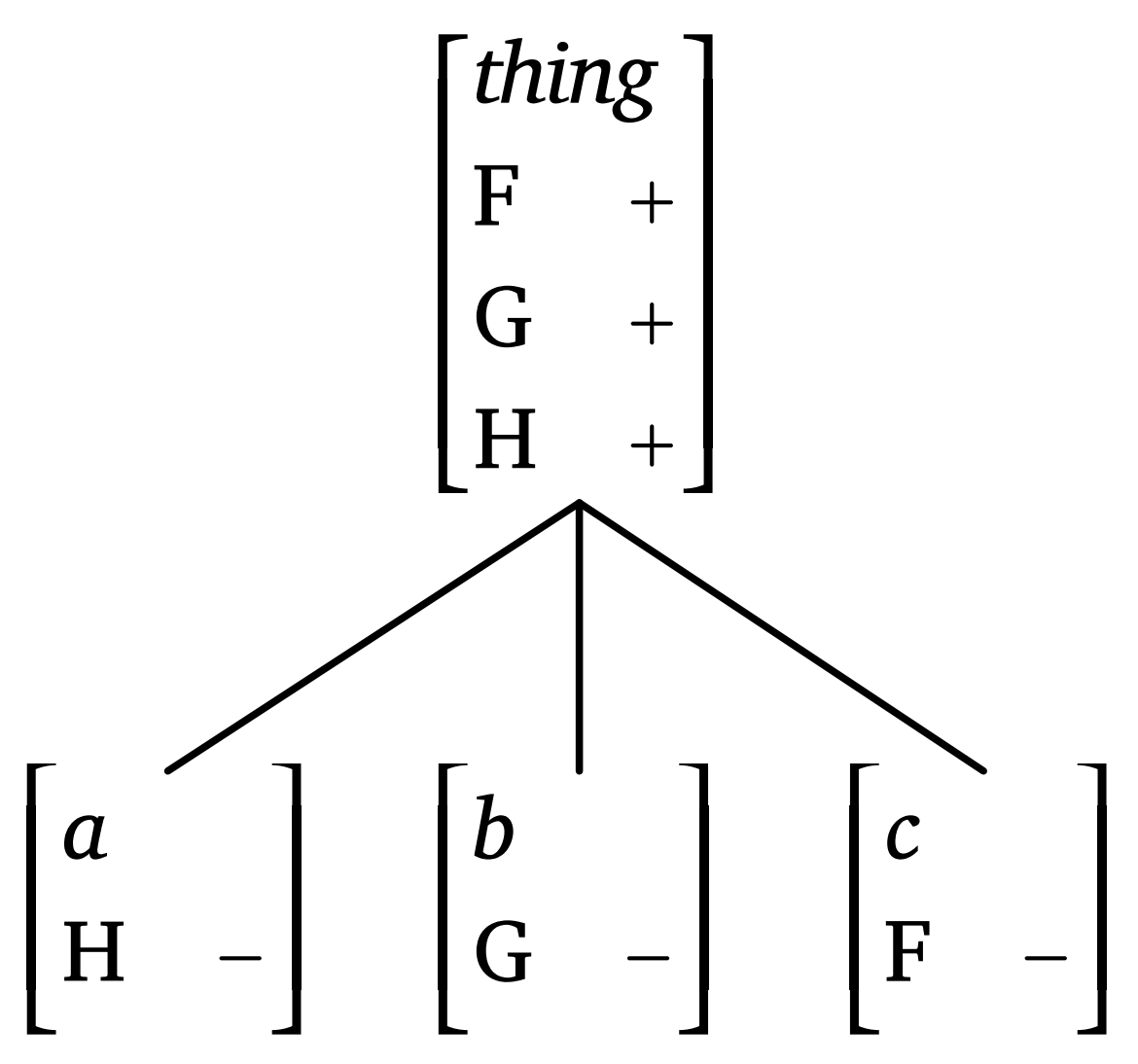




\section{Default inheritance}

Defaults give us a mechanism for representing prototypes

Once we allow overriding, what does it mean to be a member of a category?

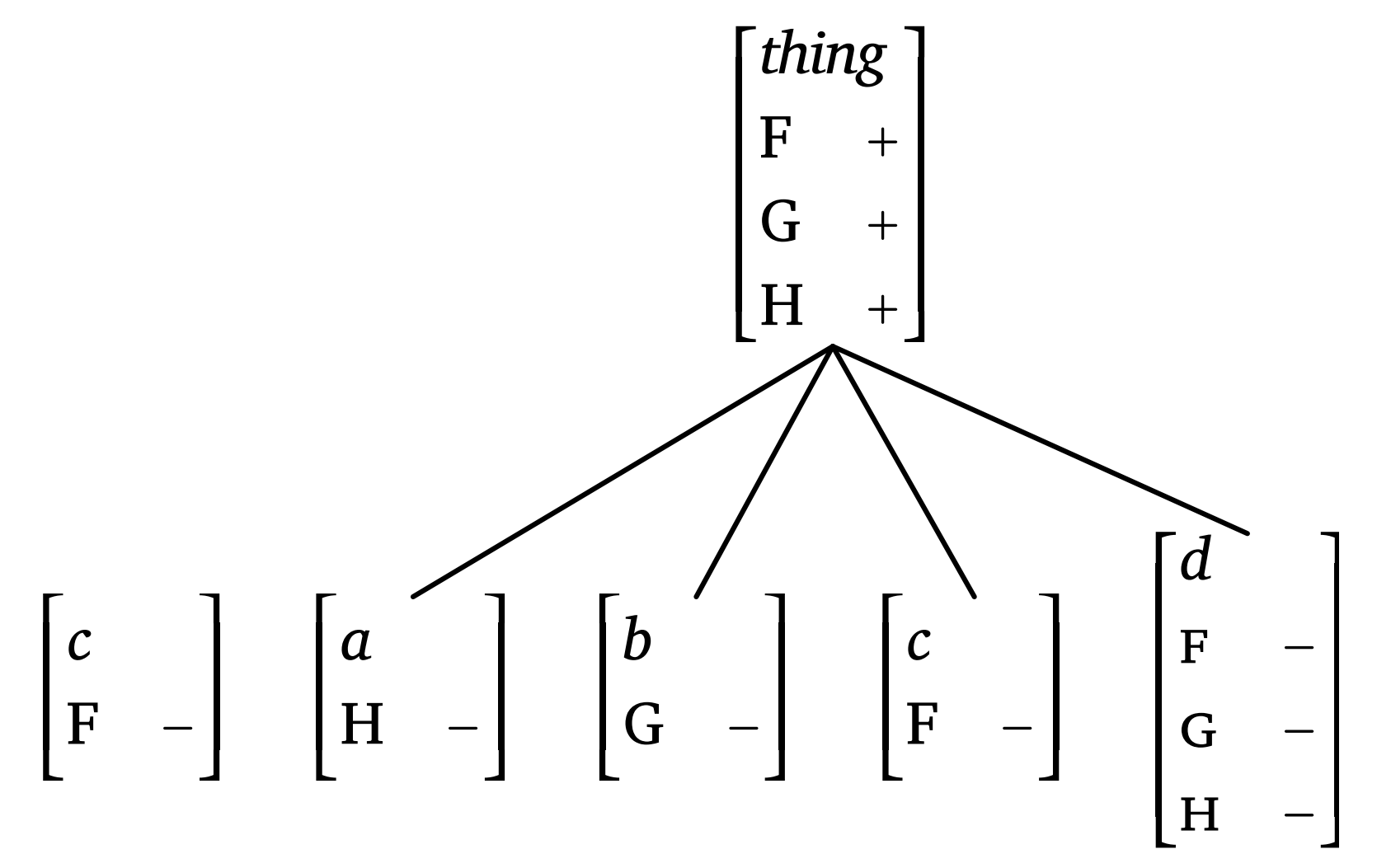

Two mechanisms for capturing similarities and differences 


\section{Prototypes}

Inheritance hierarchies (with or without overriding) come from the same knowledge representation tradition as objectoriented programming

Prototype-based programming is an alternative that has been gaining interest (Borning 1986, Lieberman 1986, Ungar and Smith 1987)

No abstract classes, only fully specified objects

All constraints are defaults

New objects are defined differentially

Objects are related to other objects via delegation 


\section{Prototypes}

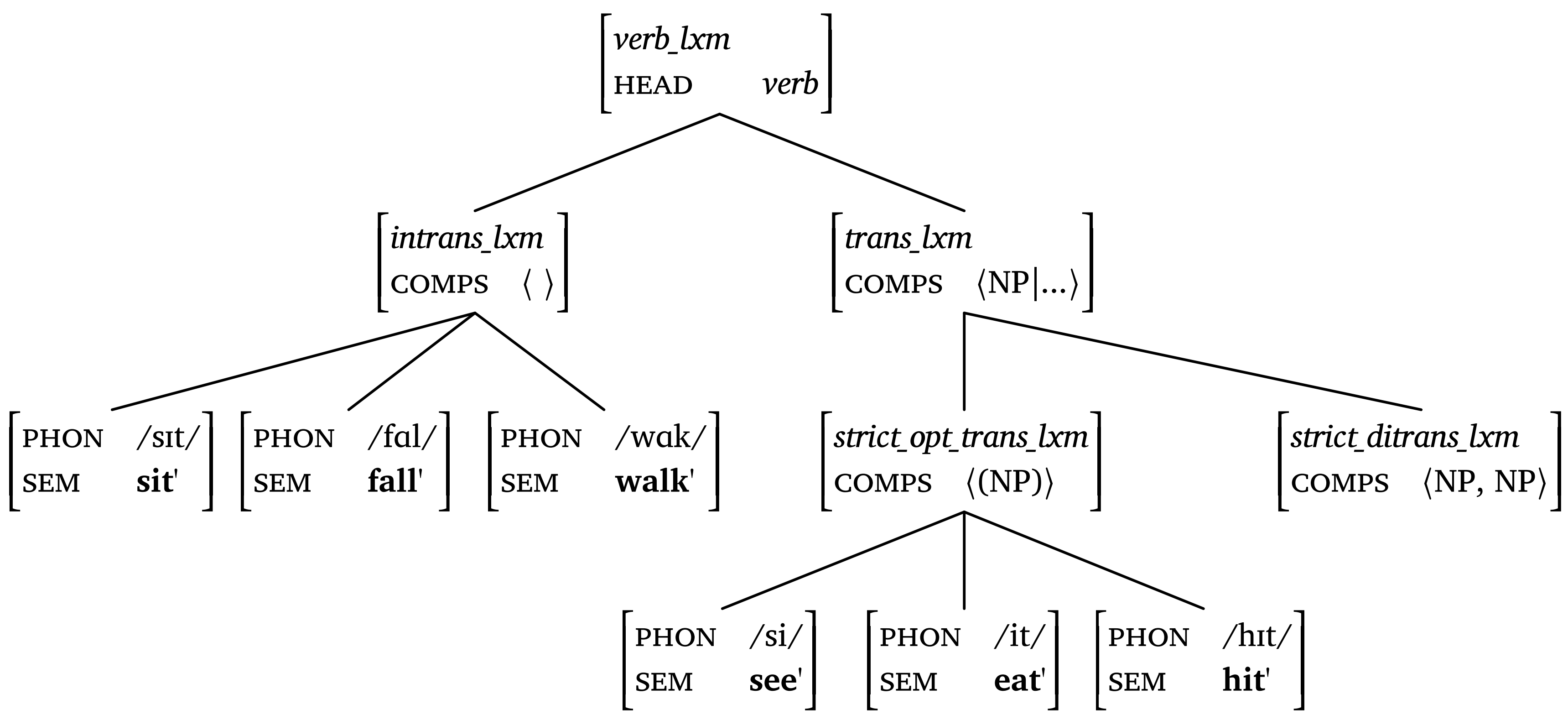




\section{Prototypes}

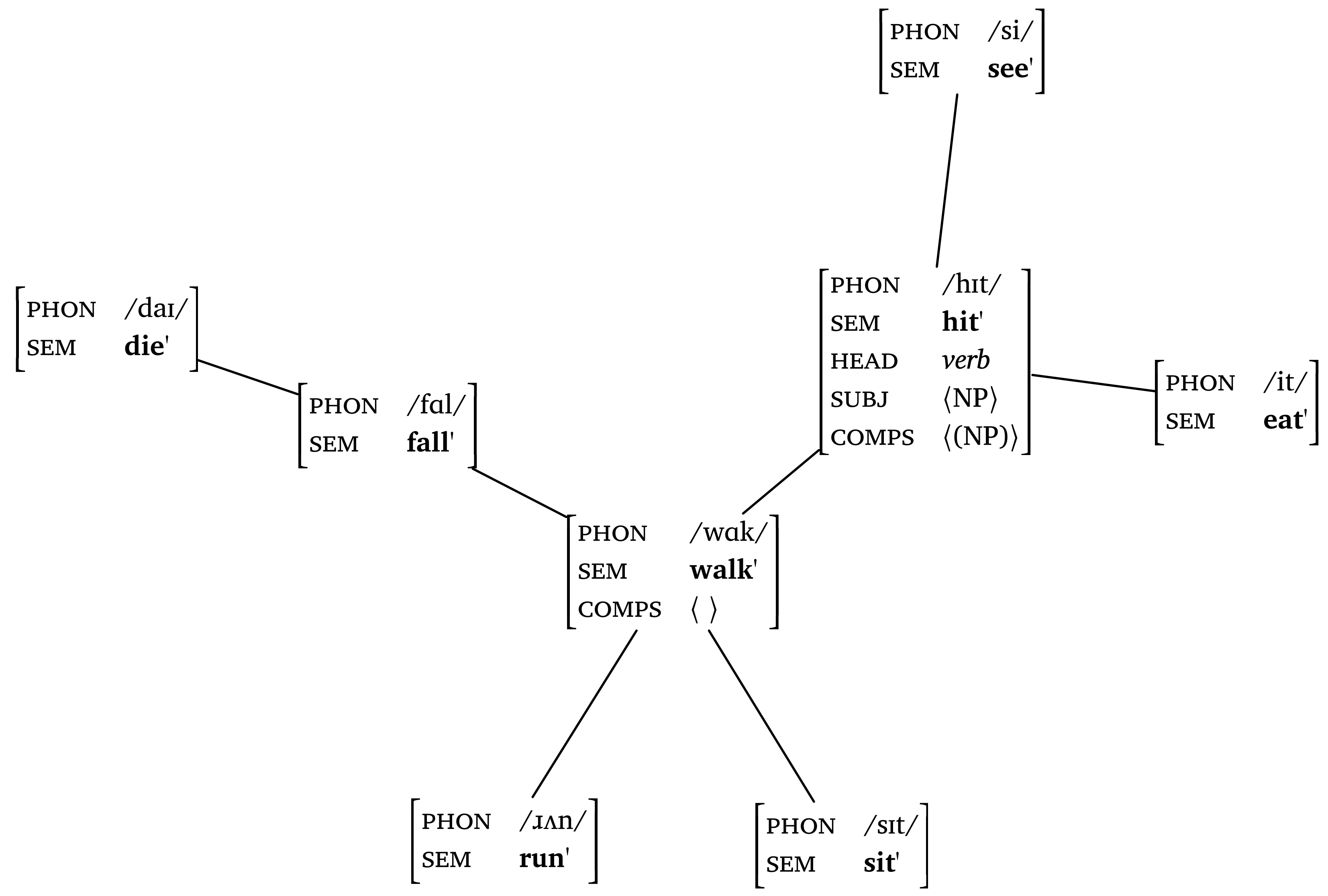




\section{Prototypes}

Inheritance

reflects an 'is-a' relation: a transitive verb is a kind of verb

default overriding is exceptionality

intensional classes and abstract prototypes

Delegation

reflects and 'is-like' relation: the lexical entry for walk is similar to the lexical entry for hit

default overriding is difference

extensional classes and concrete prototypes

Operationally, the two notions are more or less the same (Lascarides and Copestake 1999) 


\section{Prototypes}

Some obvious problems

Grammar development

Is is possible to construct and maintain differential networks like this?

Types as generalization

A taxonomic approach to the lexicon encodes the fact that there are many more verbs than there are kinds of verbs

Multiple inheritance

Words and constructions can be related to each other along multiple orthogonal dimensions 


\section{English Resource Grammar}

Large scale grammar of English (Flickinger \& Copstake 2000, Flickinger et al. 2000)

Implemented in the LKB

Organized around a large, detailed type hierarchy

Aimed at broad-coverage deep parsing and generation

Version 1111, downloaded from http://lingo.stanford.edu/ build/test/erg.tgz 


\section{English Resource Grammar}

The included lexicon (lexicon.tdl) lists 8,472 verb lexemes representing 336 types

Ten most frequent verb types account for 6,283 lexemes, and 135 verb types have only one member

$$
\begin{aligned}
& \text { v_np_le } \\
& \text { v_np*_le } \\
& \text { v_p-np_le } \\
& \text { v_p_le } \\
& \text { v_pp_e_le } \\
& \text { v_-_le } \\
& \text { v_np_noger_le } \\
& \text { v_-_unacc_le } \\
& \text { v_np-pp_e_le } \\
& \text { v_pp*_dir_le }
\end{aligned}
$$

1,723

962

896

506

494

463

408

325

322

184 


\section{English Resource Grammar}

Inverse power-law distribution (Zipf's Law)

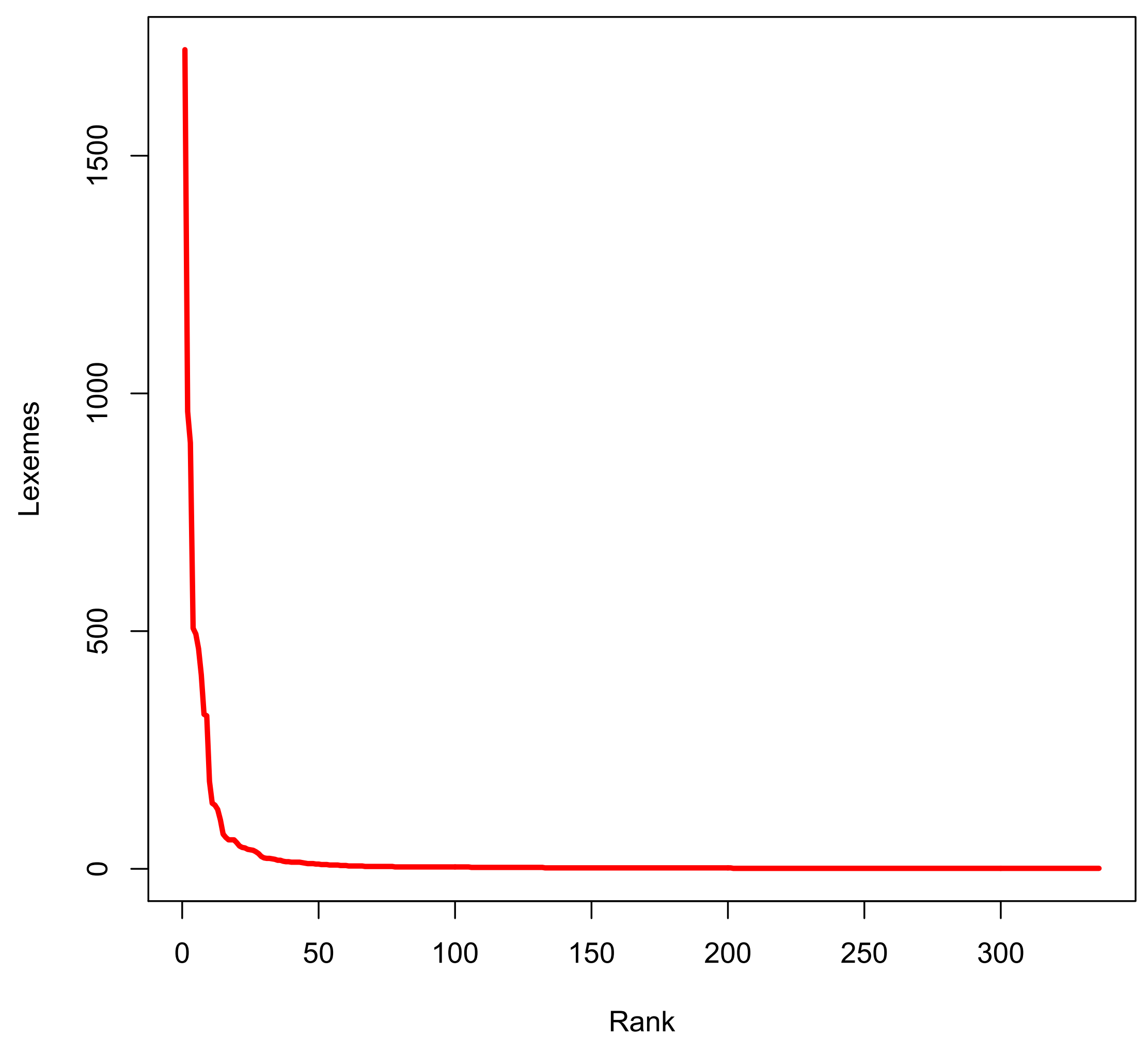




\section{English Resource Grammar}

Inverse power-law distribution (Zipf's Law)

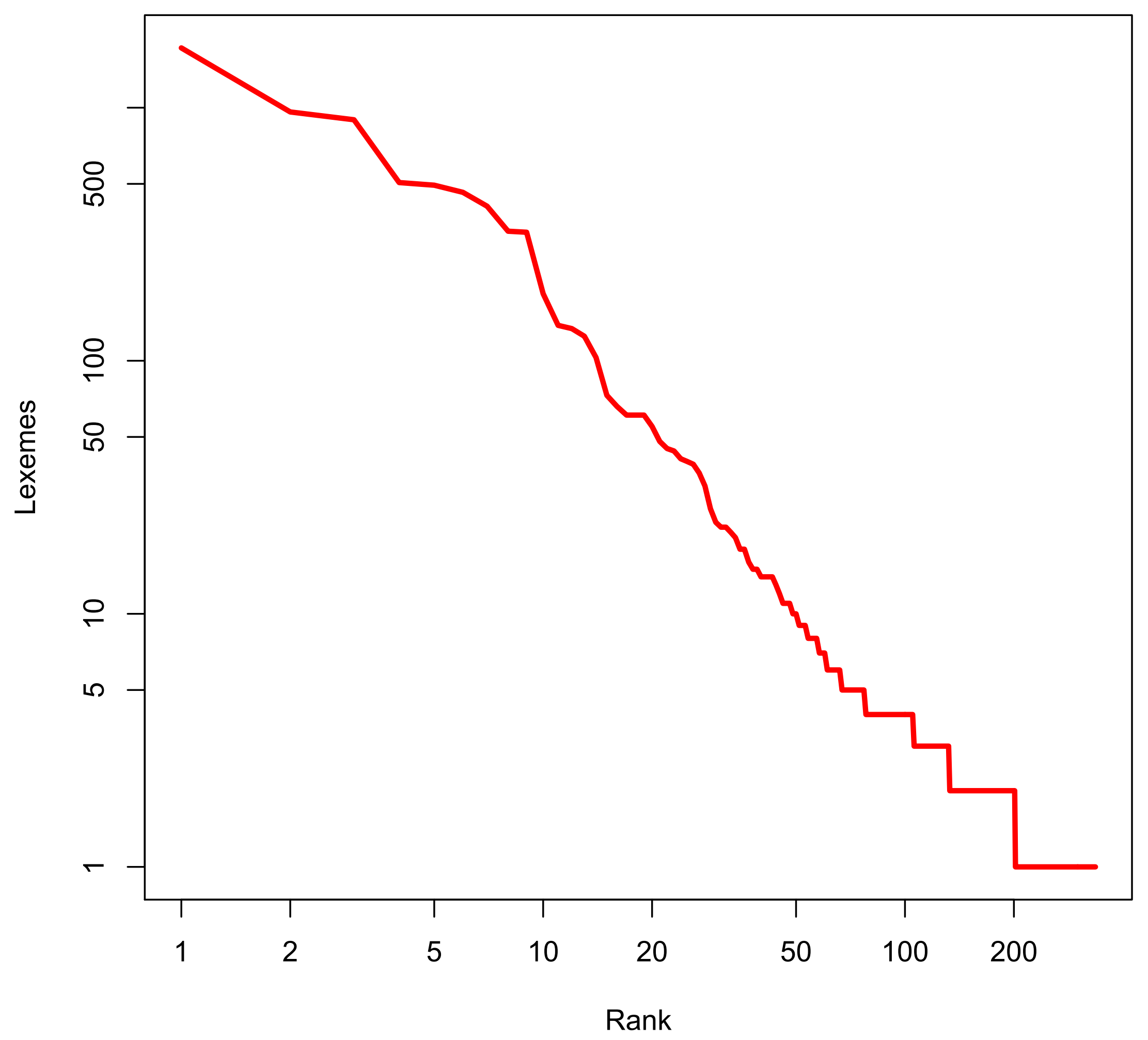




\section{English Resource Grammar}

Scale invariance: Sublexicon of 800 randomly selected verbs (96 types)

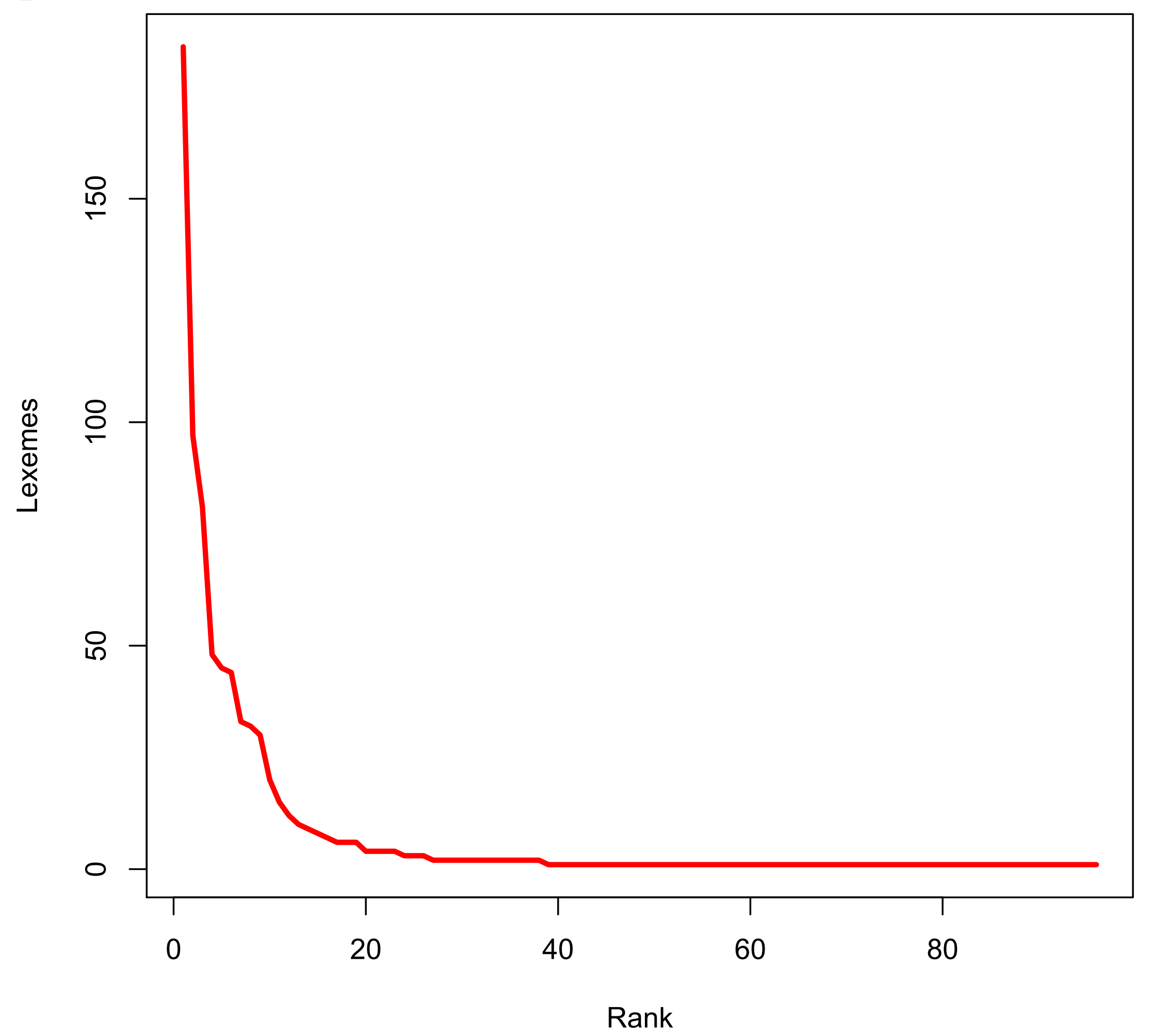




\section{English Resource Grammar}

Scale invariance: Sublexicon of 800 randomly selected verbs (96 types)

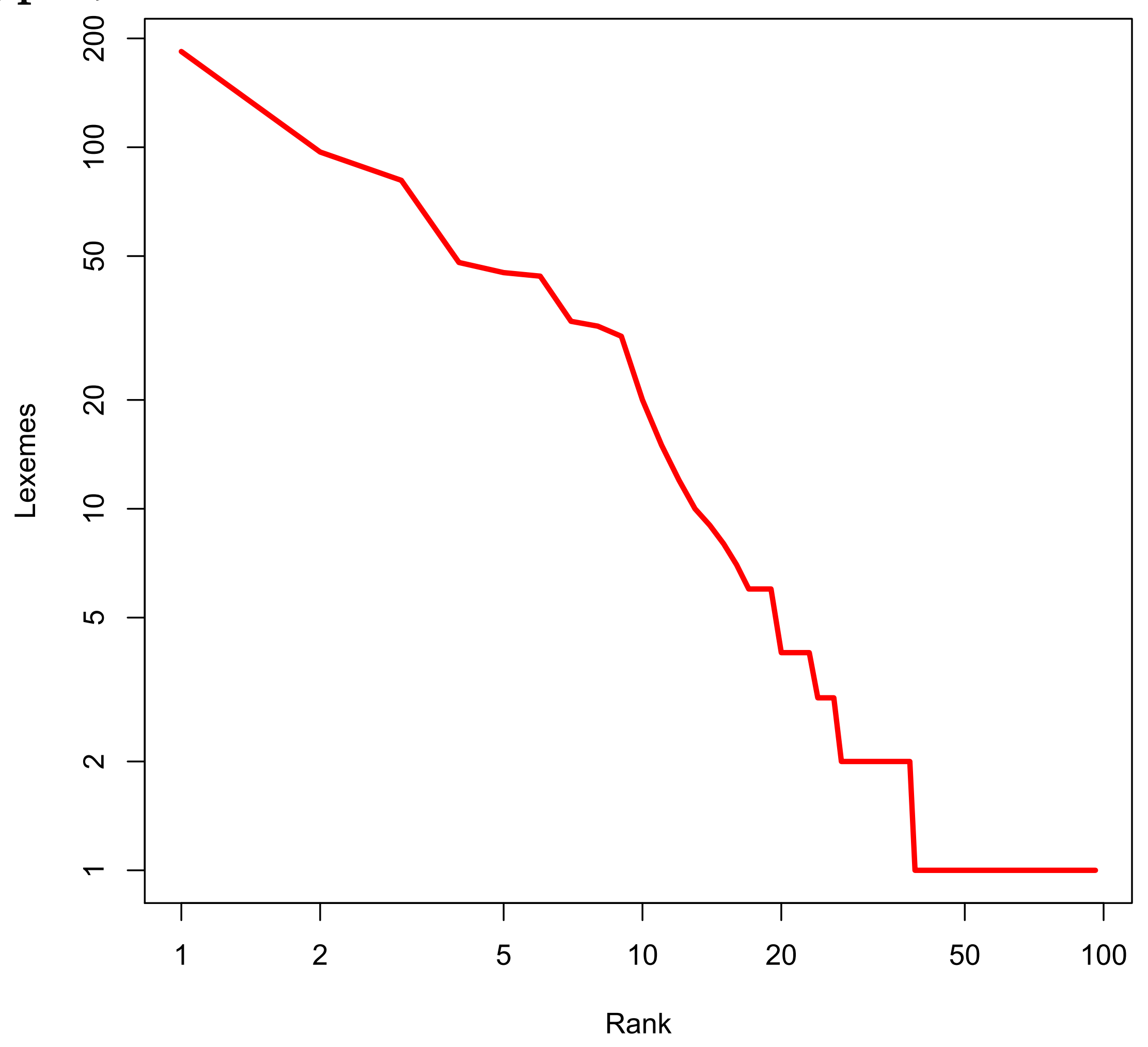




\section{British National Corpus}

The ERG covers only a small part of the English vocabulary

Even for words that are listed, entries are incomplete (Baldwin, et al. 2004)

Suppose we constructed a lexicon with $100 \%$ coverage of the BNC ... How many types would we need? 


\section{British National Corpus}

Parse each sentence using the Stanford Dependency Parser

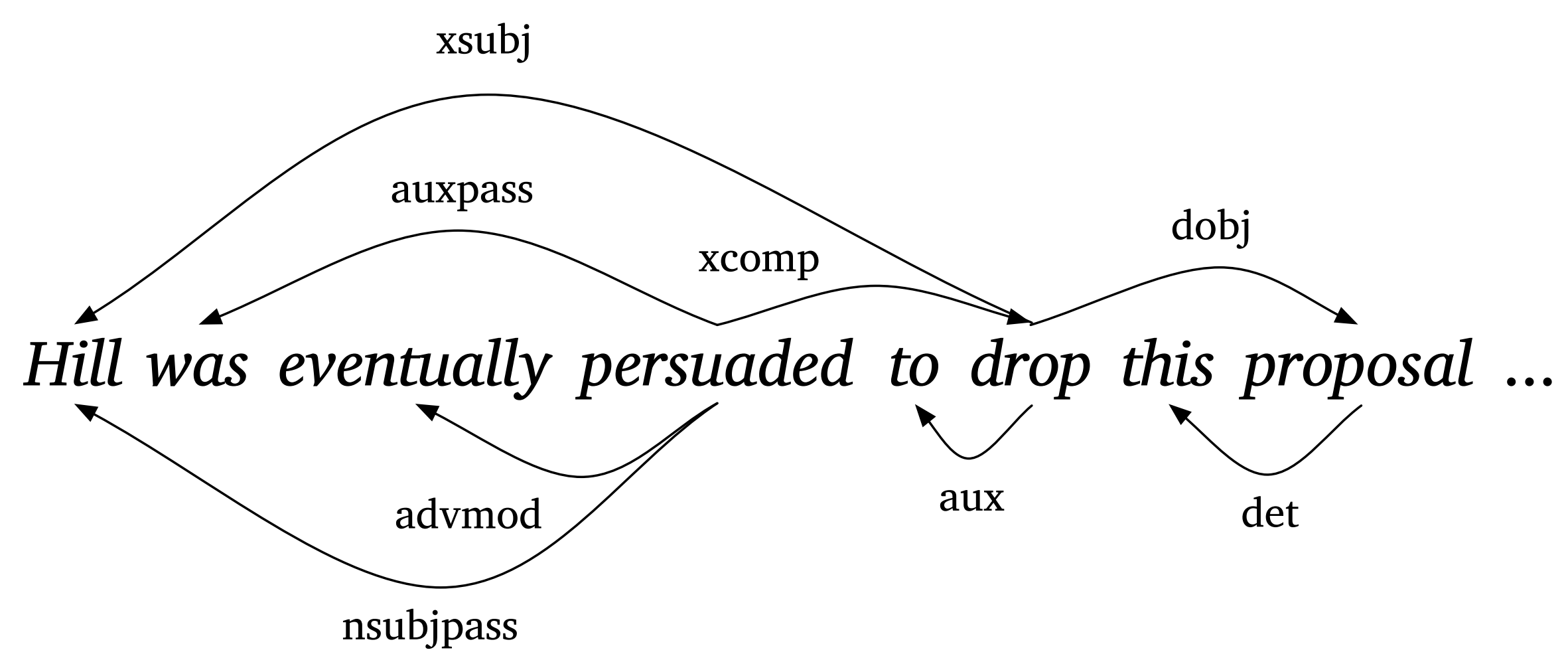

A verb frame is a bag of relations

$$
\begin{array}{ll}
\text { persuade } & \langle\text { nsubjpass, advmod, xcomp }\rangle \\
\text { drop } & \langle\text { xsubj, dobj }\rangle
\end{array}
$$




\section{British National Corpus}

A verb type is a collection of frames that a verb occurs in

\begin{tabular}{|c|c|c|c|c|}
\hline persuade & xcomp & $469 \quad$ drop & nsubj dobj & 594 \\
\hline & xsubj xcomp & 317 & nsubj dobj prep & 526 \\
\hline & nsubj xcomp & 316 & nsubj prep & 444 \\
\hline & dobj & 254 & dobj & 383 \\
\hline & dobj xcomp & 221 & prep & 275 \\
\hline & dobj ccomp & 144 & dobj prep & 266 \\
\hline & nsubjpass xcomp & 135 & nsubj dobj & 252 \\
\hline & xsubj dobj & 135 & nsubj dobj advmod & 222 \\
\hline & nsubj dobj & 126 & nsubj advmod prep & 221 \\
\hline & nsubj dobj xcomp & 112 & nsubj prep prep & 186 \\
\hline
\end{tabular}




\section{British National Corpus}

Verb frames with the highest type frequency

$\begin{array}{lr}\text { nsubj } & 15,982 \\ \text { dobj } & 13,611 \\ \text { nsubj dobj } & 13,574 \\ \text { nsubj ccomp } & 11,347 \\ \text { prep } & 9,879 \\ \text { nsubj prep } & 7,878 \\ \text { dobj prep } & 6,987 \\ \text { nsubj dobj prep } & 6,873 \\ \text { nsubj xcomp } & 5,980 \\ \text { nsubj dobj advmod } & 5,843\end{array}$




\section{British National Corpus}

Applying this method to the BNC, we get

92,612 distinct frames

67,423 verb lexemes

28,778 verb types

For each lexeme, drop frames that occur fewer than 10 times:

4,399 distinct frames

67,423 lexemes

2,554 lexical types

And if we also only consider lexemes that occur at least 500 times:

4,398 distinct frames

1,546 lexemes

1,545 lexical types 


\section{British National Corpus}

Verbs in the BNC do not appear to be organized into types

Is the lexicon structured at all?

Verb frames could be interpreted as binary features which define 'natural' classes of verbs

Or, verbs could be organized into differential network

What evidence is there for internal structure? 


\section{Spanning trees}

A delegation network is a connected acyclic graph (spanning tree) joining all lexical entries

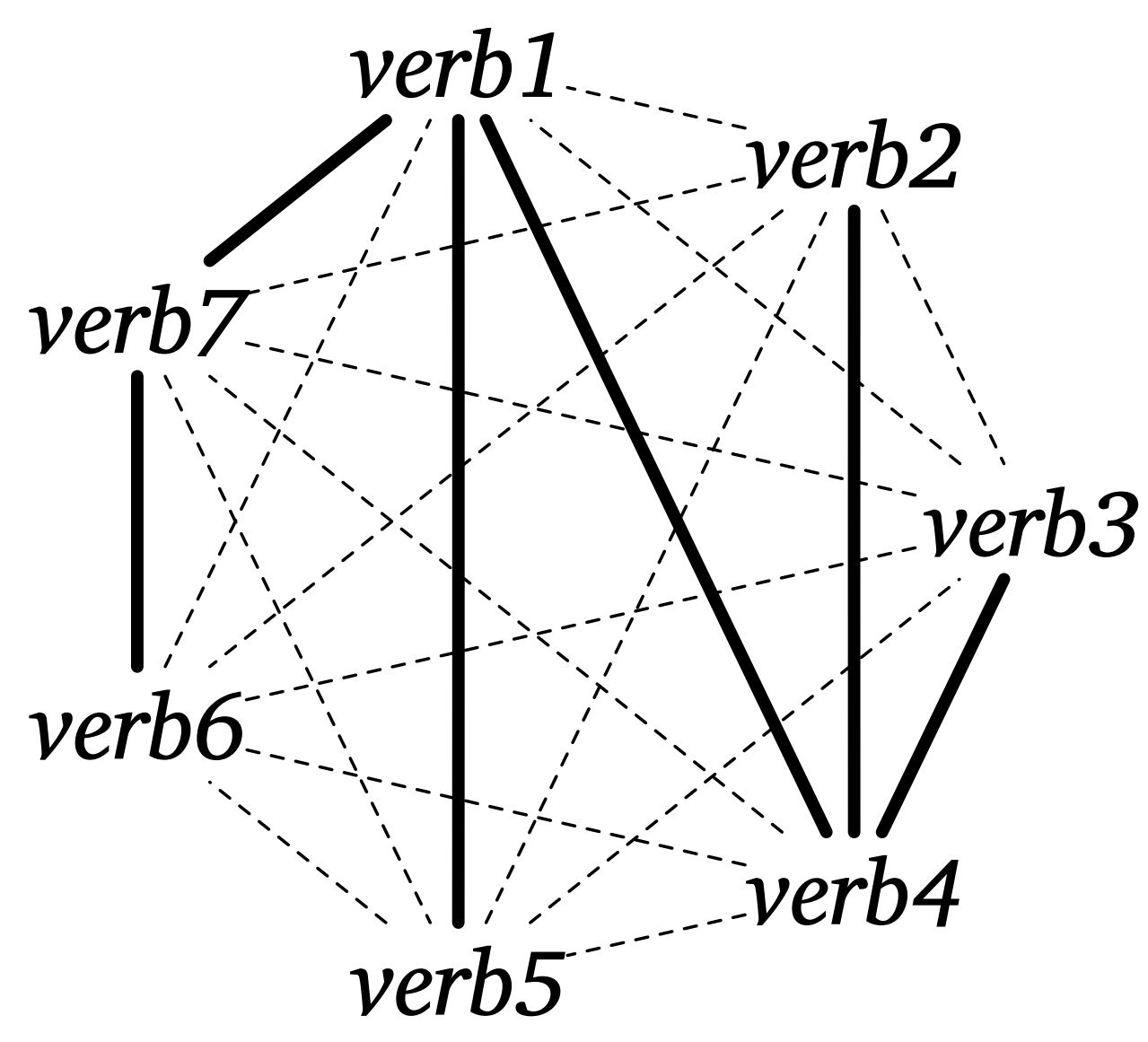

Because lexical constraints are defaults, any network structure will work - but, not all are equivalent 


\section{Spanning trees}

Evaluate networks on the basis of shared information:

Measure the difference between joined lexical entries by Jaccard distance

$$
J_{\delta}(X, Y)=1-\frac{|X \cap Y|}{|X \cup Y|}
$$

This captures the degree of default overriding between joined entries

A link between identical lexical entries would have a cost of 0

Find a minimum spanning tree - one with the smallest possible sum of edge weights (Kruskal 1956) 


\section{Spanning trees}

The minimum spanning tree cost for BNC verbs is 597.00

Is that high or low?

Generate 100 uniform random (not necessarily minimum) spanning trees (Broder 1989, Aldous 1989)

Average sum of distances is 1227.69

Min is 1216.90 and max is 1239.14

Conclusion:

There aren't many more verbs than there are types of verbs

Verbs also aren't all unique

A differential network captures at least some of the structure in the verbal lexicon 


\section{Constructions}

Ginsberg and Sag (2000) present an analysis of a range of English interrogative constructions (and other related phenomena)

Detailed syntactic and semantic model based on HPSG and (more loosely) Situation Semantics

Constructions are organized into a multiple inheritance type hierarchy with a limited degree of default overriding

Location in the hierarchy specifies a constructions syntactic and semantic properties

Sign-Based Construction Grammar (Sag 1997, van Noord et al. 1999, Sag 2007, Sag et al. 2012) 


\section{Constructions}

Declarative and interrogative constructions

\begin{tabular}{|c|c|}
\hline $\begin{array}{l}\text { decl_hd_su_cl } \\
\text { inv_decl_cl } \\
\text { decl_ns_cl } \\
\text { decl_frag_cl }\end{array}$ & $\begin{array}{l}\text { Kim smiled. } \\
\text { doesn't Kim like } \\
\text { to smile } \\
\text { Bagels. }\end{array}$ \\
\hline pol_int_cl & Did Kim leave? \\
\hline ns_wh_int_cl & What did Kim see? \\
\hline su_wh_int_cl & Who left? \\
\hline repr_int_cl & You're leaving? \\
\hline dir_is_int_cl & Kim saw Sandy? \\
\hline u_int_cl & Who? \\
\hline
\end{tabular}




\section{Constructions}

Other clause types

\begin{tabular}{|c|c|}
\hline $\begin{array}{l}\text { inv_excl_cl } \\
\text { wh_excl_cl }\end{array}$ & $\begin{array}{l}\text { Am I tired! } \\
\text { how odd it is }\end{array}$ \\
\hline ns_imp_cl & Be quiet! \\
\hline $\begin{array}{l}\text { top_cl } \\
\text { factive_cl } \\
\text { root_cl } \\
\text { cp_cl }\end{array}$ & $\begin{array}{l}\text { The bagels, I like } \\
\text { that Kim left } \\
\text { Kim left. } \\
\text { whether Kim left }\end{array}$ \\
\hline
\end{tabular}




\section{Constructions}

Non-clauses

$\begin{array}{ll}\text { fin_vp } & \text { went home } \\ \text { nf_hc_ph } & \text { going home } \\ \text { bare_nom_ph } & \text { old bagels } \\ \text { bare_adj_ph } & \text { very sad } \\ \text { nom_int_ph } & \text { who left } \\ \text { cq_np } & \text { Your name? }\end{array}$



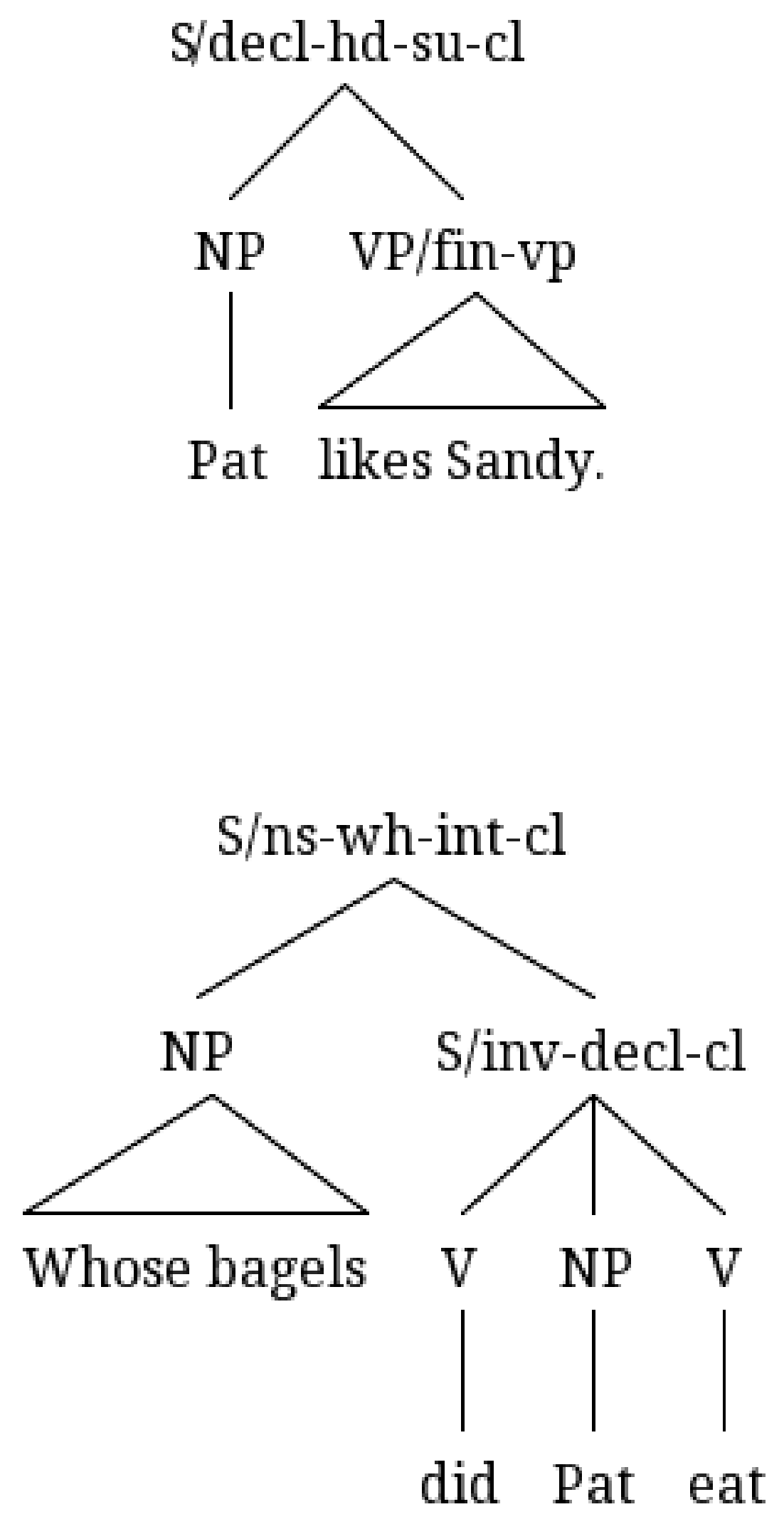
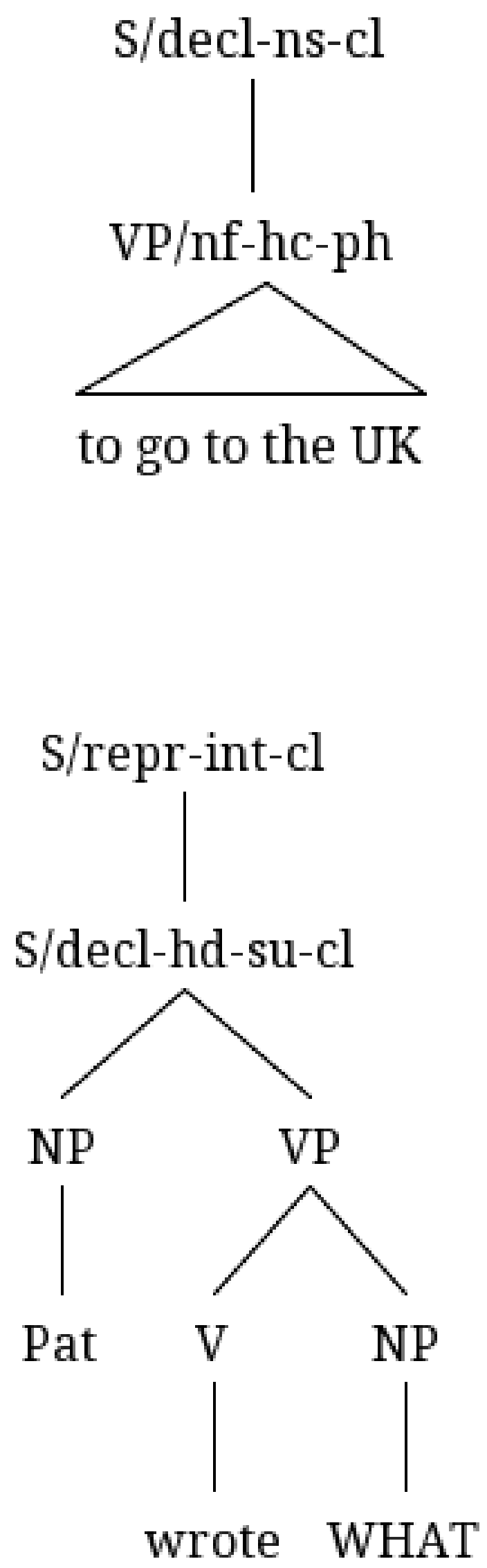


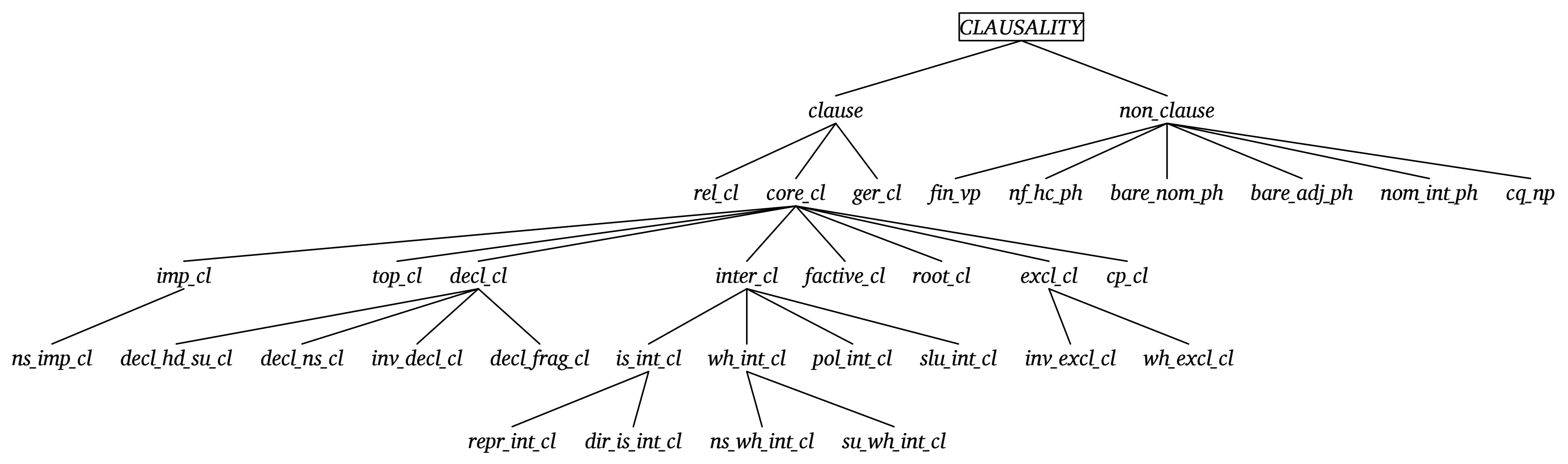




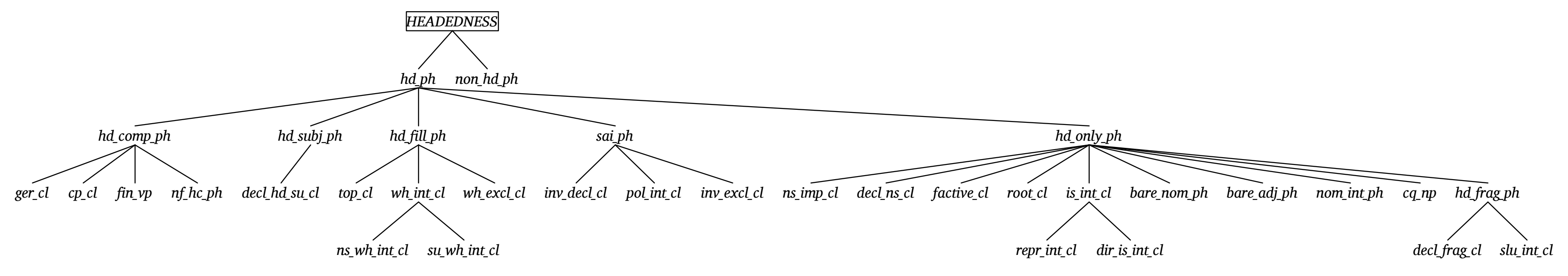




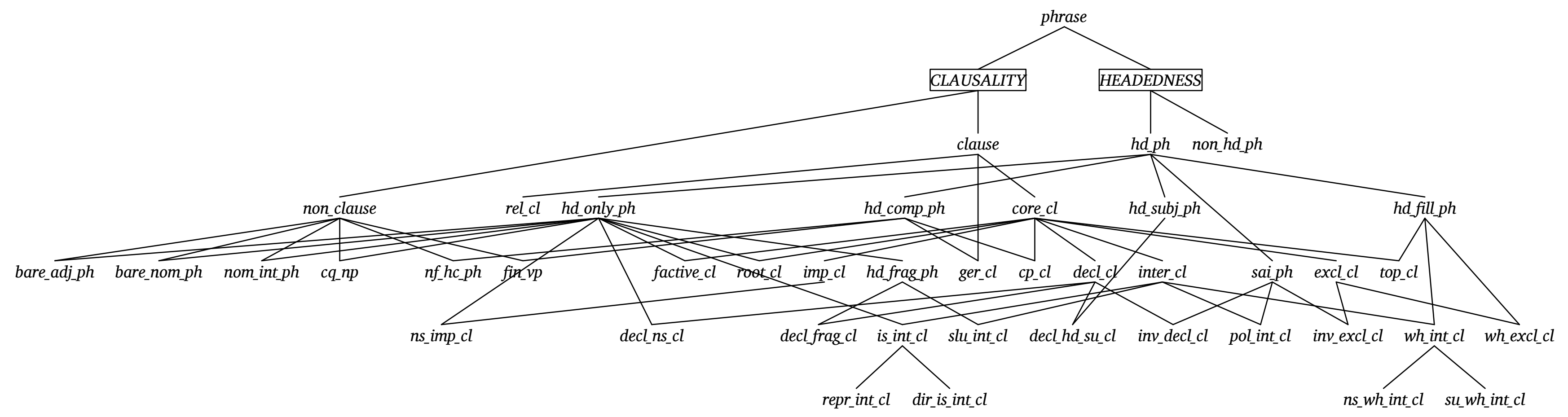




\title{
Constructions
}

\author{
Jaccard distance
}

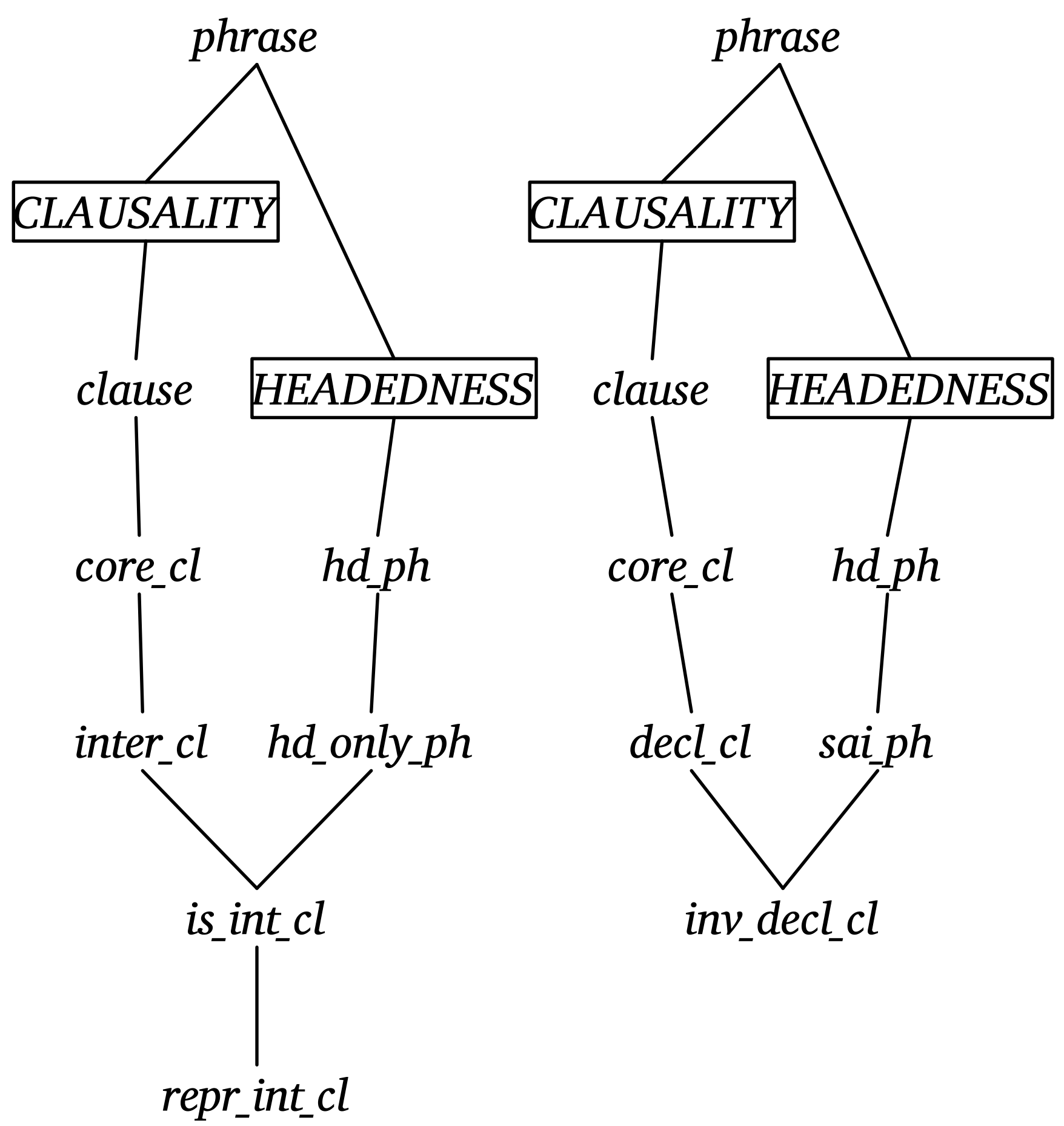




\section{Constructions}

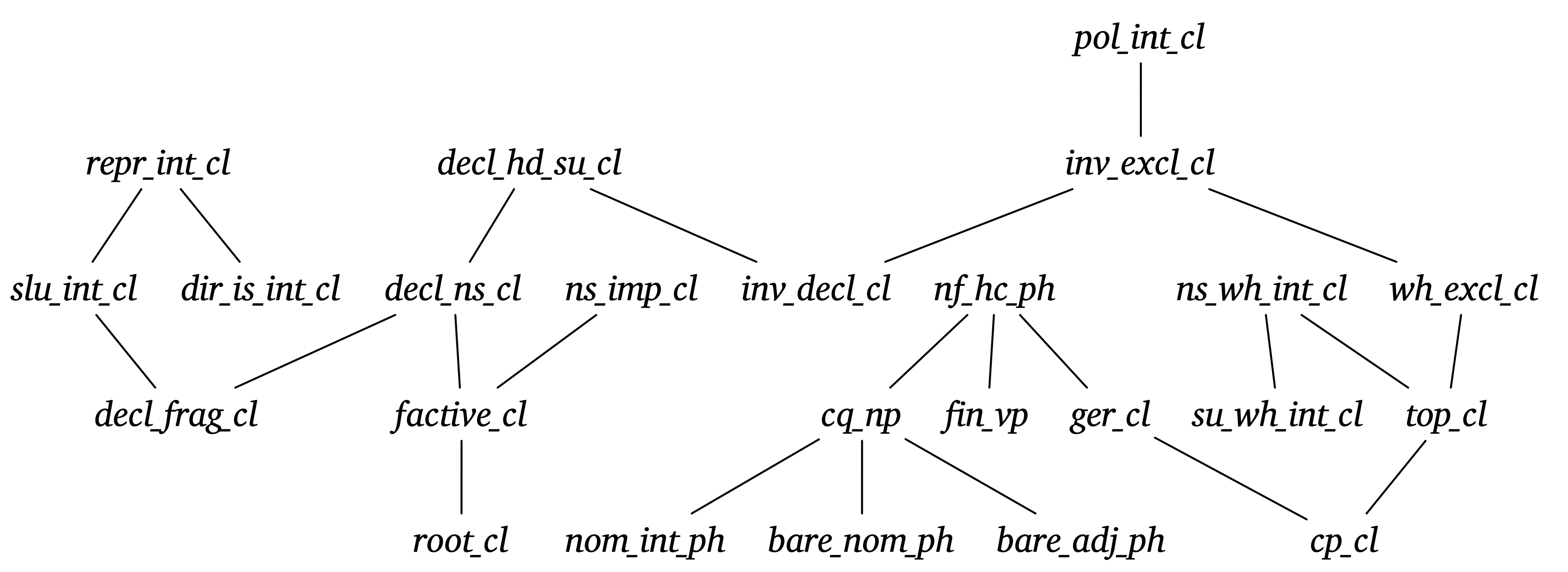




\section{Constructions}

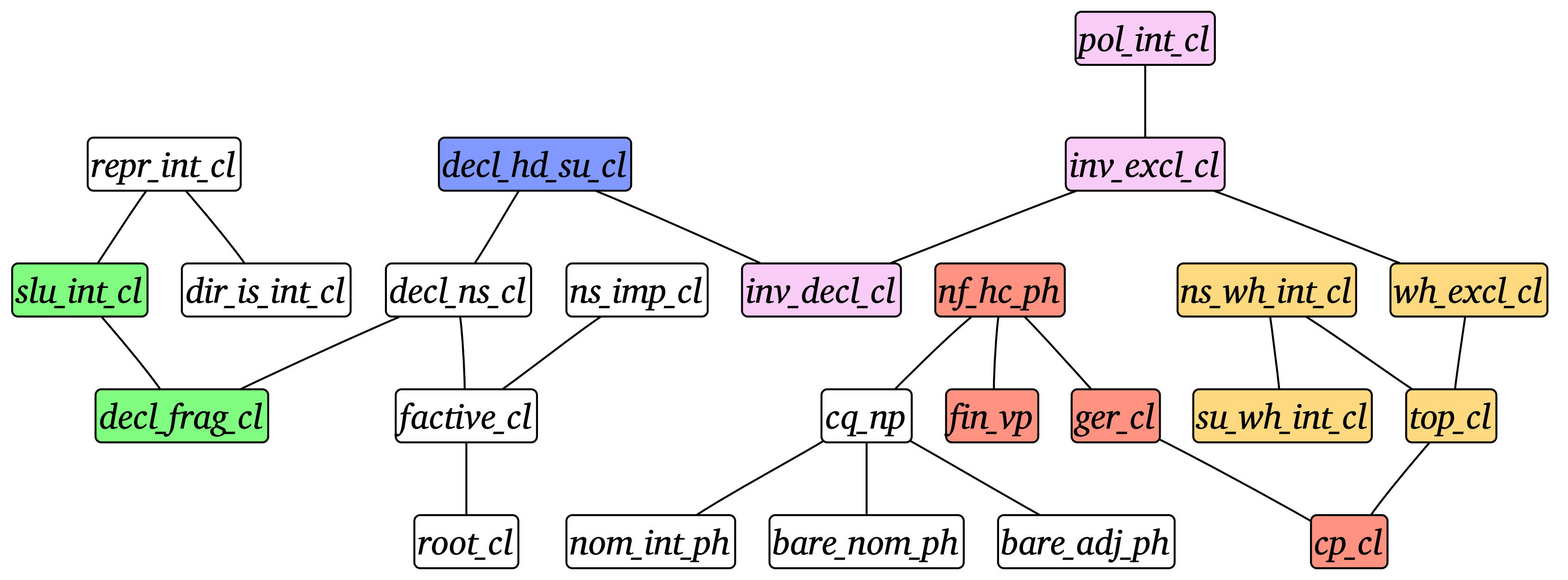

head-comp head-fill head-subj frag sai head-only 


\section{Constructions}

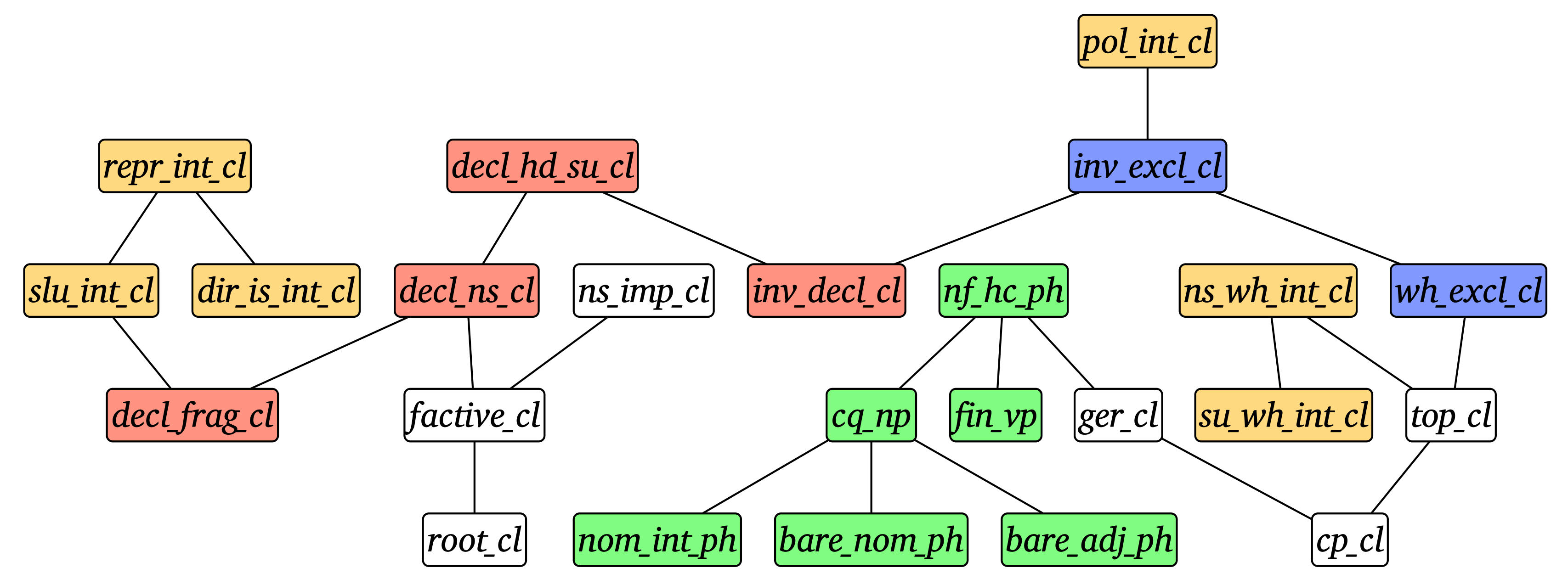

declarative interrogative exclamative non-clause other 


\section{Constructions}

The diversity is among constructions is lower than would be expected if HEADEDNESS and CLAUSALITY really were orthogonal dimensions

A flat differential network captures most (all?) of the generalizations that G\&S's complex multiple inheritance hierarchy does

Differential and hierarchical analyses aren't mutually exclusive options (cf. traits)

Approaching the problem of organizing constructions quantitatively may reveal patterns that aren't otherwise obvious 


\section{Prospects}

Differential networks are a viable alternative to taxonomic representations

How far can they be extended?

Richer datasets

Other lexicalist frameworks (Network Morphology, Word Grammar)

How can they be refined?

Families as a step towards types (Astudillo and Schilling 1993)

No reason to limit focus to spanning trees (Ackerman and Bonami)

Types, tokens, exemplars (Abbot-Smith and Tomasello 2006, Baayen et al. 2007) 